\title{
Vegetation dynamics in Qinling-Daba Mountains in relation to climate factors between 2000 and 2014
}

\author{
LIU Xianfeng ${ }^{1,2}$, ZHU Xiufang ${ }^{1,2}$, "PAN Yaozhong ${ }^{1,2}$, LI Shuangshuang ${ }^{1,3}$, \\ MA Yuqi ${ }^{2}, \mathrm{Nie} \mathrm{Juan}^{4}$ \\ 1. State Key Laboratory of Earth Surface Processes and Resource Ecology, Beijing Normal University, Beijing \\ 100875, China; \\ 2. College of Resources Science \& Technology, Beijing Normal University, Beijing 100875, China; \\ 3. Academy of Disaster Reduction and Emergency Management, Beijing Normal University, Beijing 100875, \\ China: \\ 4. National Disaster Reduction Center of China, Beijing 100124, China
}

\begin{abstract}
Using the Moderate Resolution Imaging Spectroradiometer-normalized difference vegetation index (NDVI) dataset, we investigated the patterns of spatiotemporal variation in vegetation coverage and its associated driving forces in the Qinling-Daba (Qinba) Mountains in 2000-2014. The Sen and Mann-Kendall models and partial correlation analysis were used to analyze the data, followed by calculation of the Hurst index to analyze future trends in vegetation coverage. The results of the study showed that (1) NDVI of the study area exhibited a significant increase in 2000-2014 (linear tendency, 2.8\%/10a). During this period, a stable increase was detected before 2010 (linear tendency, 4.32\%/10a), followed by a sharp decline after 2010 (linear tendency, -6.59\%/10a). (2) Spatially, vegetation cover showed a "high in the middle and a low in the surroundings" pattern. High values of vegetation coverage were mainly found in the Qinba Mountains of Shaanxi Province. (3) The area with improved vegetation coverage was larger than the degraded area, being $81.32 \%$ and $18.68 \%$, respectively, during the study period. Piecewise analysis revealed that $71.61 \%$ of the total study area showed a decreasing trend in vegetation coverage in 2010-2014. (4) Reverse characteristics of vegetation coverage change were stronger than the same characteristics on the Qinba Mountains. About $46.89 \%$ of the entire study area is predicted to decrease in the future, while $34.44 \%$ of the total area will follow a continuously increasing trend. (5) The change of vegetation coverage was mainly attributed to the deficit in precipitation. Moreover, vegetation coverage during La Nina years was higher than that during El Nino years. (6) Human activities can induce ambiguous effects on vegetation coverage: both positive effects (through implementation of ecological restoration projects) and negative effects (through urbanization) were observed.
\end{abstract}

Keywords: vegetation coverage; spatiotemporal variation; attribution analysis; ENSO; Qinling-Daba (Qinba) Mountains

Received: 2015-08-03 Accepted: 2015-08-30

Foundation: Major Project of High-resolution Earth Observation System; Beijing Natural Science Foundation, No.8144052

Author: Liu Xianfeng (1986-), PhD Candidate, specialized in resource and environmental remote sensing and disaster remote sensing. E-mail: liuxianfeng7987@163.com

"Corresponding author: Pan Yaozhong (1965-), PhD and Professor, specialized in statistics and disaster remote sensing research. E-mail: pyz@bnu.edu.cn 


\section{Introduction}

The Qinling-Daba (Qinba) Mountains are an important geoecological boundary in central China and the water source for the middle route of South-to-North Water Diversion Project and the largest continuous poverty areas in China. Hence it is of great significance for the region to sustainable development of both economy and ecological environment. As many problems have emerged in the global population, resources, and environment since the 20th century, sustainable development of human beings faces great challenges ( $\mathrm{Fu}, 2014)$, and the terrestrial ecosystem has been greatly disturbed by climate change and human activities. As an ecologically vulnerable area that is extremely sensitive to climate change, the ecosystem of the Qinba Mountains may be changed or even damaged under rapid climate change (Walther et al., 2002; Parmesan, 2006). However, little is known about the ecosystem of the area because of limited and fragmented data, and an overall survey and evaluation of the main geographical features are required to improve our understanding of the effect of climate change on typical terrestrial ecosystems ${ }^{1}$. As the main factor of a terrestrial ecosystem, vegetation is significantly affected by climate change, and in turn, it influences the atmospheric environment to a great extent through feedback of biochemical processes. Moreover, vegetation coverage is widely recognized as a priority indicator in monitoring the ecological environment and evaluating ecological risk and vulnerability (Liu et al., 2013). Thus, it is important to analyze spatiotemporal variation in vegetation coverage on the Qinba Mountains in order to understand the influence of climate and human factors on its ecosystem.

With increasing concern over global climate change, there is much interest in the relationship between the terrestrial ecosystem and global climate change (Walker et al., 1997; Shi et al., 2014); the response of vegetation to climate change is being studied by scholars worldwide (Nemani et al., 2003; Ma et al., 2012). According to previous studies, climate change can be divided into trend, fluctuation, and extreme events (Stocker et al., 2013; Shi et al., 2014). Many scholars have ascribed the variation in vegetation coverage to the former two aspects of climate change (Zhang et al., 2013; Ding et al., 2007), while there are fewer studies on the influence of extreme climate events on vegetation growth. Recently, scholars worldwide have evaluated the response of vegetation to extreme climate conditions in key ecological zones, such as Amazon and Congo rainforests, and have drawn conclusions (Hilker et al., 2014; Lewis et al., 2011; Zhou et al., 2014). However, in China, there are few studies in this area, especially studies of regions extremely vulnerable to climate change, like the Qinba Mountains. According to IPCCAR5, atmospheric temperature has globally increased by $0.72^{\circ} \mathrm{C}\left(0.49-0.89^{\circ} \mathrm{C}\right)$ on average in the past 60 years (1951-2012) (Stocker et al., 2013), while it has increased by $1.38^{\circ} \mathrm{C}$ in China between 1960 and 2009. This suggests that the speed of temperature increase is higher in China than globally and in the Northern Hemisphere (Compiling Committee, 2011); the greatest increase was observed in the northern part of China, resulting in a growth trend of the frequency and intensity of extreme events (Liu et al., 2014; Reichstein et al., 2013). This increased the risk and vulnerability of terrestrial ecosystems and endangered ecological security. In addition, other studies have suggested that the correlation between vegetation growth and atmospheric temperature is

\footnotetext{
${ }^{1}$ Presentation of academicians Sun Honglie, Zheng Du, and Sun Jiulin at a seminar conference on the environment and development of the Qinba Mountains and rivers and their impacts on arid and semi-arid regions.
} 
weakening and that drought is probably the reason (Piao et al., 2014). Thus, extreme climate events influence not only vegetation but also the relationship between vegetation and climate features. Since there is a significant difference in the structure of geographical features and their changing patterns in different regions, focusing on a regional study is more beneficial for understanding the evolution of terrestrial ecosystems (Fu, 2014). Thus, it is necessary to re-evaluate vegetation coverage and response to the climate because recent climate change has caused vegetation to be exceptionally sensitive to extreme weather conditions.

Considering that the Qinba Mountains are a key ecological zone, studying its geographical features by now still weak. Moreover, since extreme climate events are occurring more frequently because of recent great climate fluctuations, it is urgent to study the relation between extreme climate and vegetation coverage change for the smooth implementation of the projects for synthetic risks prevention and disaster reduction and control. Meanwhile, the initiation of the "New-Round Grain for Green Project Plan", acquiring a complete understanding the background feature of vegetation and its response to climate change has become very important. Therefore, we analyzed the current trend, fluctuation, and predicted future trend of vegetation coverage on the Qinba Mountains by using the normalized difference vegetation index (NDVI) data and trend analysis, residual analysis, and the Hurst exponent method. Through analyzing the changing trend in vegetation coverage, we can find the variation characteristics of the regional environment, facilitating the synergetic development of regional ecology and social economy.

\section{Data and methods}

\subsection{Study area}

The Qinba Mountains, located in central China between $102^{\circ} 54^{\prime}-112^{\circ} 40^{\prime} \mathrm{E}$ and $30^{\circ} 50^{\prime}-$ $34^{\circ} 59^{\prime} \mathrm{N}$ (Figure 1), has a total area of $222,300 \mathrm{~km}^{2}$ and across Shaanxi, Gansu, Sichuan, Hubei, Henan, and Chongqing (Bai, 2014). They are divided into three units on the basis of topographical features: Qinling Mountains, Hanjiang River valley, and Daba Mountains. Since Qinling Mountains and Daba Mountains both help in blocking cold air from the north and warm air from the south-west and the region is controlled by Mongolian cold high in

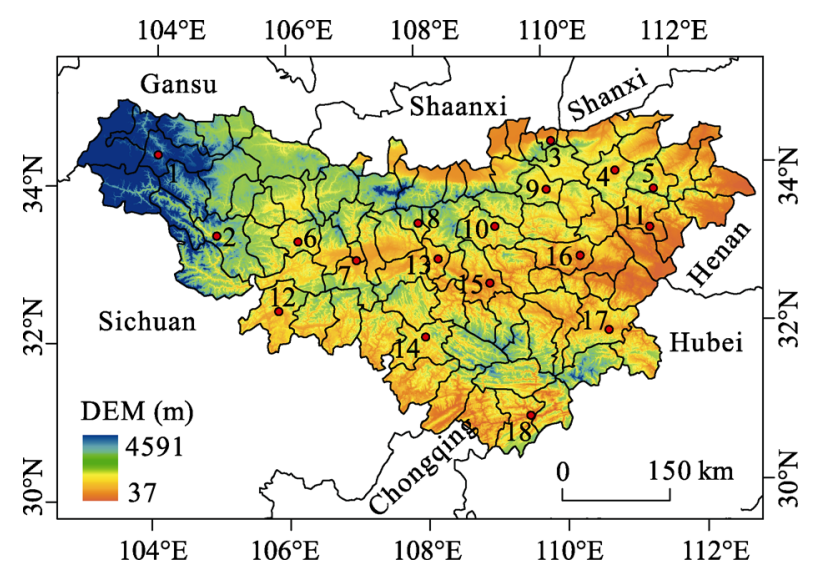

Figure 1 Study area and distribution of meteorological stations in the Qinba Mountains 
winter and by both west-extended Pacific subtropical high and Sichuan Basin warm low in summer, the climate is cold and dry in winter and wet with summer drought in summer across the study area (Liu, 1983). The vegetation is highly diversified because of a climate transition of the subtropical zone and warm temperate zone, with the north and south biotas converging together. In Qinling Mountains, warm temperate deciduous broad-leaved forests are dominantly found, and to the south of the region, a mixed broad-leaved deciduous forests and evergreen broad-leaved forests are found (Ren et al., 2003).

\subsection{Data source}

Remote sensors were used to monitor vegetation coverage, mainly on the basis of spectral reflection information and the contrast between strong absorption in the visible spectrum and high reflection in the near-infrared spectrum (Jiang et al., 2008). Hundreds of vegetation indices have been proposed on the basis of these characters, and the normalized difference vegetation index (NDVI) is most widely used because it effectively responds to vegetation fluctuation (Barati et al., 2011). Considering that the study area is a mountain region, we selected the MOD13Q1NDVI dataset obtained from EOS/MODIS product of NASA to analyze vegetation coverage change in the study area because of the high spatial resolution of $250 \mathrm{~m} \times 250 \mathrm{~m}$ and time resolution of 16 days. The time span of NDVI used in our study was from 2000 to 2014. The dataset was guaranteed to be of high quality and dealt with water, clouds, and heavy aerosol, and it is widely used in studies on regional vegetation coverage. First, data form and projection of the raw MODIS-NDVI data were transformed using MODIS Reprojection Tools. Then, we acquired monthly NDVI data by using the max value composite to eliminate the effect of outliers (Holben, 1986). Finally, we calculated maximum annual NDVI, which reflects the true situation of vegetation coverage in a region, to detect spatiotemporal variation in NDVI.

The meteorological datasets (18 meteorological stations in the Qinba Mountains in 2000-2014), including average temperature, maximum temperature, minimum temperature, relative humidity, sunshine duration, wind speed, and precipitation, were obtained from the China Meteorological Data Sharing Service System (http://cdc.cma.gov.cn). Southern oscillation index (SOI) data for the same period were obtained from 74 circulation index datasets published by the national climate center on climate change and prediction research associated with the China Bureau of Meteorology.

\subsection{Methods}

\subsubsection{Trend analysis}

The trend of NDVI in the study area was calculated using Sen trend analysis (Sen, 1968), and the significance of the trend was further examined using the Mann-Kendall statistical test (Kendall, 1948). The advantage of Sen trend analysis is that the method does not require a specific sample distribution and is free from the interference of outliers. Therefore, this method is robust and resistant to errors due to measurements and outlier data. The formula is as follows:

$$
\beta=\operatorname{mean}\left(\frac{x_{j}-x_{i}}{j-i}\right) \quad \forall j>i
$$


where $\beta$ represents the trend of NDVI; $i$ and $j$ denote the time series, and $x_{i}$ and $x_{j}$ denote the NDVI value at time $i$ or $j$, respectively. $\beta>0$ suggests that the vegetation coverage increases, and $\beta<0$ suggests otherwise.

\subsubsection{Stability analysis}

Coefficient of variation (CV) can be used to describe the relative fluctuation in geographical data (Xu, 2002). Thus, we adopted CV to reflect the stability of NDVI change. The formula for $\mathrm{CV}$ is as follows:

$$
C_{v}=\frac{1}{x} \sqrt{\frac{\sum_{i=1}^{n}\left(x_{i}-\bar{x}\right)^{2}}{n-1}}
$$

where $C_{v}$ is the $\mathrm{CV} ; i$ is time series; $x_{i}$ is the NDVI value at time $i$, and $\bar{x}$ denotes the average of NDVI during the study period. A higher $C_{v}$ value denotes the existence of a large fluctuation in the time series and vice versa.

We further analyzed the fluctuation in NDVI by using residual analysis, in order to obtain more robust results. Specifically, (1) calculate the residual values of NDVI according to the regression model, (2) use the absolute value of the residual to generate a residual time series, and (3) perform regression analysis of the residual time series (the value of the trend is approximated to zero, which suggests less fluctuations in NDVI and vice versa).

\subsubsection{Future trend analysis}

The Hurst exponent, proposed by the British hydrologist Hurst, is estimated using R/S analysis and is an effective way of measuring the long-time dependence of a time series. The main principles are as follows (John et al., 2008).

1. Given a time series $\{\xi(t)\} t=1,2, \ldots, n$, divide the time series into $\tau$ subseries $\xi(t)$.

2. Define the mean sequence of the time series:

$$
\langle\xi\rangle_{\tau}=\frac{1}{\tau} \sum_{t=1}^{\tau} \xi(t) \quad \tau=1,2,
$$

3. Calculate cumulative deviation:

$$
X(t, \tau)=\sum_{u=1}^{t}\left(\xi(u)-\langle\xi\rangle_{\tau}\right) \quad 1 \leqslant t \leqslant \tau
$$

4. Calculate range sequence:

$$
R(\tau)=\max _{1 \leqslant t \leqslant \tau} X(t, \tau)-\min _{1 \leqslant t \leqslant \tau} X(t, \tau) \quad \tau=1,2, \ldots
$$

5. Calculate standard deviation sequence:

$$
S(\tau)=\left[\frac{1}{\tau} \sum_{t=1}^{\tau}\left(\xi(t)-\langle\xi\rangle_{\tau}\right)^{2}\right]^{\frac{1}{2}} \tau=1,2, \ldots
$$

If $R / S \propto \tau^{H}$, the time series shows the Hurst phenomenon, and the $H$ value is called the Hurst exponent, which can be obtained by least squares fitting in the double logarithmic coordinate system. According to Hurst (1951), the value of the Hurst exponent expands from 0 to 1 , and it can be divided into three groups: (1) $H>0.5$ refers to the persistence of the series, which indicates the same trend in the time series in the future, with a greater value for more persis- 
tence. (2) $H=0.5$ implies that the time series was random without persistence, which indicates that changes in the time series in the future would be unrelated to those in the study period. (3) $H<0.5$ refers to anti-persistence of the time series, which indicates an anti-trend in the time series in the future, with smaller values for more anti-persistence sustainability.

\section{Results}

\subsection{Temporal variations of vegetation coverage}

Spatial averaged NDVI showed a significant increasing trend in the Qinba Mountains between 2000 and 2014, with a linear tendency of 2.8\%/10a $(p<0.001)$. Piecewise analysis showed that the variation trend of NDVI reversed in 2010. Vegetation coverage was con-

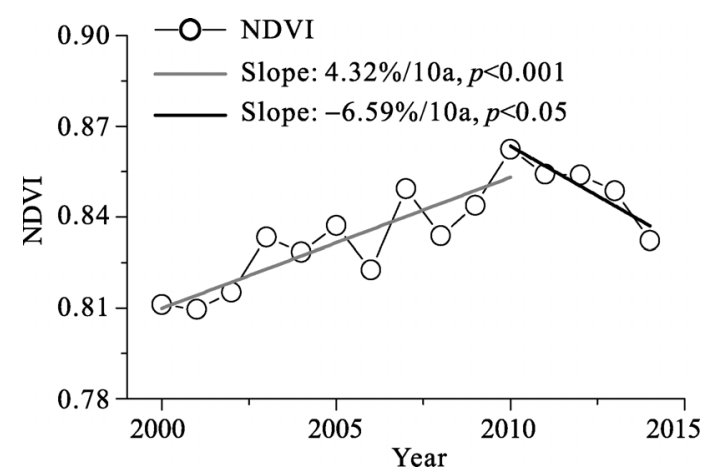

Figure 2 Temporal variation in vegetation coverage in the Qinba Mountains during 2000-2014 tinuously growing at the rate of $4.32 \% / 10 \mathrm{a}$ before $2010(p<0.001)$, and it declined sharply at $6.59 \% / 10 \mathrm{a}(p<0.05)$ afterwards; in 2014, it decreased to the greatest extent (Figure 2). This phenomenon suggested that despite ecological restoration projects, like the large-scale Grain for Green project, in the area, vegetation coverage did not grow stably after 2010, but showed a growing trend at first and a decreasing trend afterwards; this may be attributed to the largescale drought observed recently.

\subsection{Spatial variations of vegetation coverage}

\subsubsection{General characteristics of NDVI}

Generally, vegetation coverage is high in the central part of the region and low at the edges. High values of NDVI were mainly distributed in the Qinling and Daba Mountains of Shaanxi Province because these regions are covered by well-grown broad-leaved forests, coniferous forests, and shrubs. Low values of NDVI were distributed in Lixian, Xihe, and Wudu of Longnan; Wushan and Gangu of Tianshui; Zhouqu of south Gansu; and some parts of the Hanjiang river valley, mostly because these areas are mainly croplands with low NDVI. However, vegetation coverage in these areas is rapidly recovering because of the Grain for Green Project. According to the NDVI frequency plot, vegetation coverage is generally high in the Qinba Mountains, and areas where NDVI is greater than 0.7 account for $92.48 \%$ of the whole region (Figure $3 \mathrm{a}$ ). With increasing altitude, the vegetation coverage on the Qinba Mountains is gradually growing. Vegetation coverage between 500 and $3600 \mathrm{~m}$ is stable, and the highest at $1200 \mathrm{~m}$. However, it decreases rapidly after $3600 \mathrm{~m}$, probably because only grasslands and shrubs are found at a low density at this height (Figure 3b).

\subsubsection{Trend of NDVI change}

To analyze the variation trend in vegetation coverage in the Qinba Mountain region, we calculated the trend of NDVI between 2000 and 2014 and the result was classified into 4 

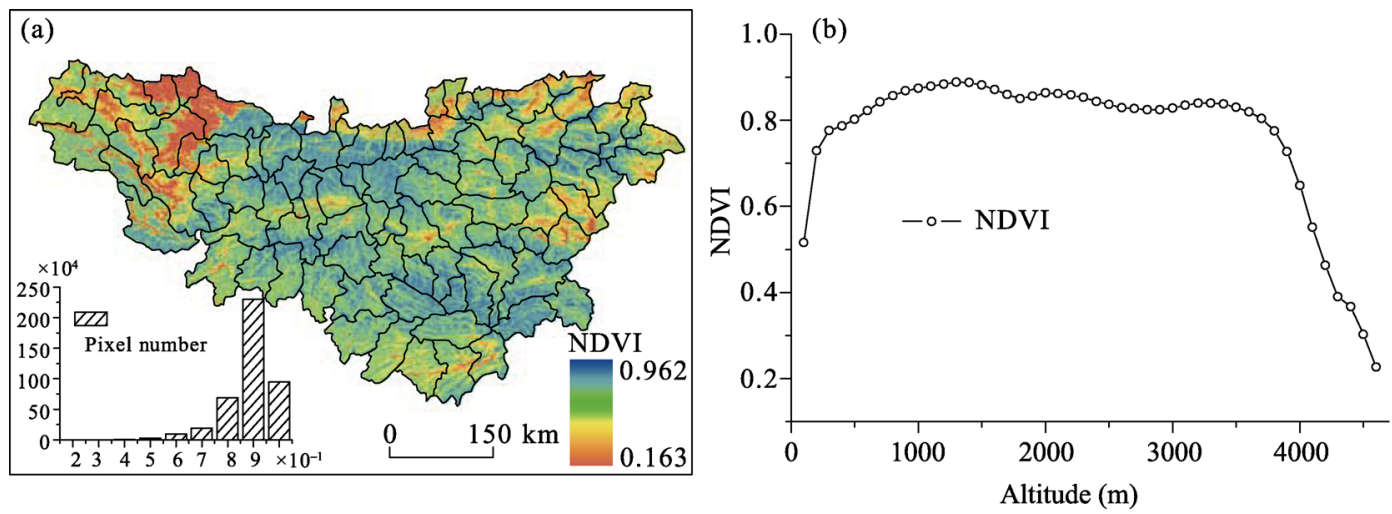

Figure 3 Spatial distribution (a) and altitude-dependent (b) of NDVI on the Qinba Mountains

levels after the M-K test: extremely significant variation $(p<0.01)$, significant variation $(p<$ $0.05)$, weakly significant variation $(p<0.1)$, and no significant variation. Results show that, generally, vegetation coverage in the region increased. The area with improved vegetation coverage was larger than the degraded area, being $81.32 \%$ and $18.68 \%$, respectively, during the study period. As for the former part, $46.86 \%$ shows no obvious variation, while extremely significant increased and significant increased areas account for $7.18 \%$ and $16.14 \%$, respectively, of the entire study area (Table 1). Spatially, areas with significant vegetation coverage growth were mainly located in the northwestern part of the region because of the implementation of the Grain for Green Project (Figure 4). Further analysis of the variation trend between 2010 and 2014 showed that NDVI values decreased in $71.61 \%$ of the whole Qinba Mountain region, of which significant variation and extremely significant variation accounted for $6.38 \%$ and $3.45 \%$, respectively, being mainly distributed in eastern Longnan, southwestern Baoji, all counties in Hanzhong, and southwestern Ankang.

Table 1 Area and proportion of different vegetation change types on the Qinba Mountains

\begin{tabular}{lcccccc}
\hline \multicolumn{1}{c}{ Type } & \multicolumn{3}{c}{$2000-2014$} & & \multicolumn{2}{c}{$2010-2014$} \\
\cline { 2 - 7 } & $\begin{array}{c}\text { Pixel } \\
\text { number }\end{array}$ & $\begin{array}{c}\text { Percent- } \\
\text { age/\% }\end{array}$ & $\begin{array}{c}\text { Cumulative } \\
\text { percentage/\% }\end{array}$ & $\begin{array}{c}\text { Pixel } \\
\text { number }\end{array}$ & $\begin{array}{c}\text { Percentage } \\
/ \%\end{array}$ & $\begin{array}{c}\text { Cumulative } \\
\text { percentage/\% }\end{array}$ \\
\hline $\begin{array}{l}\text { Decrease } \\
\text { Weakly significantly } \\
\text { decrease }\end{array}$ & 795821 & 18.68 & 18.68 & 2384471 & 55.96 & 55.96 \\
$\begin{array}{l}\text { Significantly increase } \\
\begin{array}{l}\text { Extremely signifi- } \\
\text { cantly decrease }\end{array}\end{array}$ & - & - & - & 248378 & 5.83 & 61.78 \\
$\begin{array}{l}\text { Increase } \\
\begin{array}{l}\text { Weakly significantly } \\
\text { increase }\end{array}\end{array}$ & - & - & - & 271746 & 6.38 & 68.16 \\
$\begin{array}{l}\text { Significantly increase } \\
\begin{array}{l}\text { Extremely signifi- } \\
\text { cantly increase }\end{array}\end{array}$ & 674747 & 11.14 & 76.67 & 46298 & 1.09 & 71.61 \\
\hline
\end{tabular}

\subsubsection{Stability of NDVI change}

The coefficient of variation for 2000-2014 was between 0.004 and 0.84 , suggesting significant spatial differences in the stability of vegetation coverage in the study area. Specifically, 

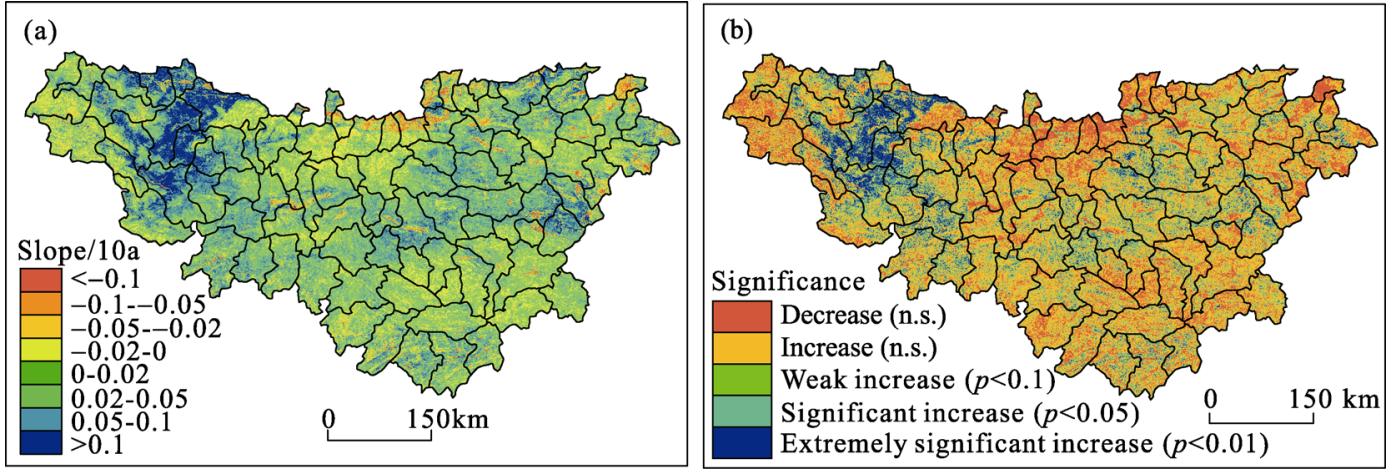

Figure 4 Trend and significance of NDVI on the Qinba Mountains in 2000-2014

areas with a low value were located mainly on the Qinling and Daba Mountains of Shaanxi Province, where broad-leaved forests and coniferous forests account for most of the vegetation and the variation is relatively stable. Areas with a high value were mainly located in the northwestern and eastern parts, where the land cover is arable land. Because of ecological restoration projects like Grain for Green, vegetation coverage in these areas is recovering at an unstable pace (Figure 5a). Furthermore, a residual analysis trend close to 0 also confirmed the low value of the variation coefficient (Figure 5b). Together, residual analysis trend and variation coefficient results were used in cross-validation for testing the credibility of this study.
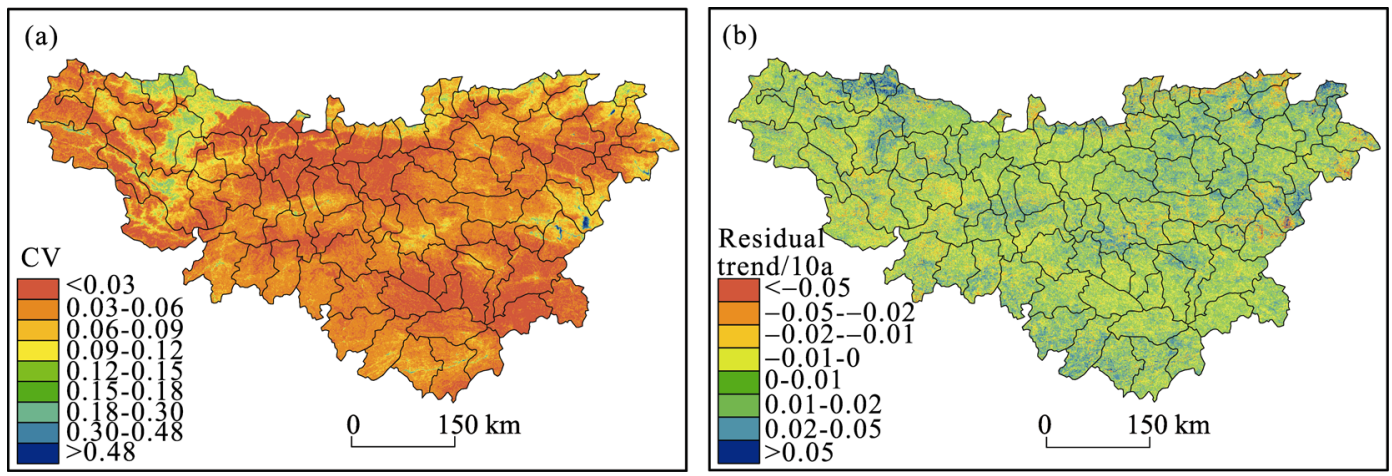

Figure 5 Trend and significance of NDVI on the Qinba Mountains in 2000-2014

\subsection{Future trend of NDVI}

The above mentioned analysis mainly focused on the spatiotemporal variation of NDVI on the Qinba Mountains for 15 years; however, the trend of NDVI in the future is still unclear. Therefore, we calculated the future trend of NDVI on the basis of the Hurst exponent (Figure 6a). Results show that the average Hurst exponent is 0.4857 (0.0996-0.9837). Pixels where the Hurst exponent is less than 0.5 account for $57.12 \%$, which indicates that the reverse characteristics of vegetation coverage change were stronger than the same characteristics on the Qinba Mountains. Statistical analysis showed that $46.89 \%$ of the entire study area is predicted to decrease from increase and $8.44 \%$ is predicted to degrade continuously in future, while $34.44 \%$ of the total area will follow a continuously increasing trend. Mean- 
while, areas where the Hurst exponent between 0.4 and 0.6 account for $68.15 \%$ and areas where the Hurst exponent is less than 0.4 and 0.6 account for $19.36 \%$ and $12.49 \%$, respectively, indicating that a relatively small area exhibited the strong reverse and same characteristics. It should be noted that the Hurst exponent varies slowly with an increase in altitude. While the value is mostly less than 0.5 under $4500 \mathrm{~m}$, it increases significantly afterwards; this suggests that areas less than $4500 \mathrm{~m}$ mainly showed reverse characteristics (Figure 6b).
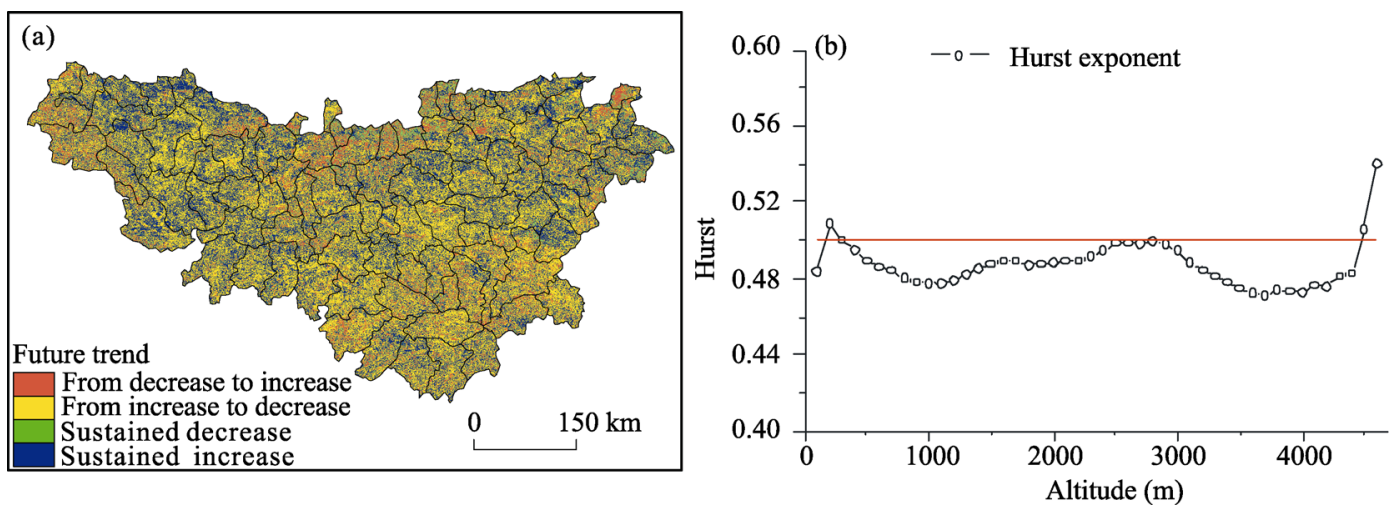

Figure 6 Future trend of NDVI and its difference with altitude on the Qinba Mountains

\section{Attribution analysis of vegetation change}

\subsection{Response of vegetation coverage to climate factors}

Global climate change has altered the regional pattern of temperature and precipitation, and therefore influenced vegetation growth. Statistical analysis showed that both temperature and precipitation showed a weakly increasing trend between 2000 and 2013 at the speed of $0.15^{\circ} \mathrm{C} / 10 \mathrm{a}(p=0.56)$ and $32.06 \mathrm{~mm} / 10 \mathrm{a}(p=0.66)$, respectively. Precipitation at Lueyang and Hanzhong in Shaanxi (stations 6 and 7) and Guangyuan in Sichuan (Station 12) has increased significantly, while precipitation showed a significantly decreasing trend at Fengjie in Chongqing (Station 18), which is consistent with a sharp increase in temperature and intensifies the magnitude of water deficit in this region (Figure 7).

To ensure the accuracy of the data and enhance the reliability of the research results, we adopted site-by-site partial correlation analysis to explore the response of vegetation to climate factors. Details of this method are as follows: (1) first, the NDVI values were extracted within a boundary of $3 \mathrm{~km} \times 3 \mathrm{~km}$ according to the meteorological sites. (2) The partial correlation coefficient between NDVI and temperature and precipitation was calculated using detrend NDVI values, temperature, and precipita-

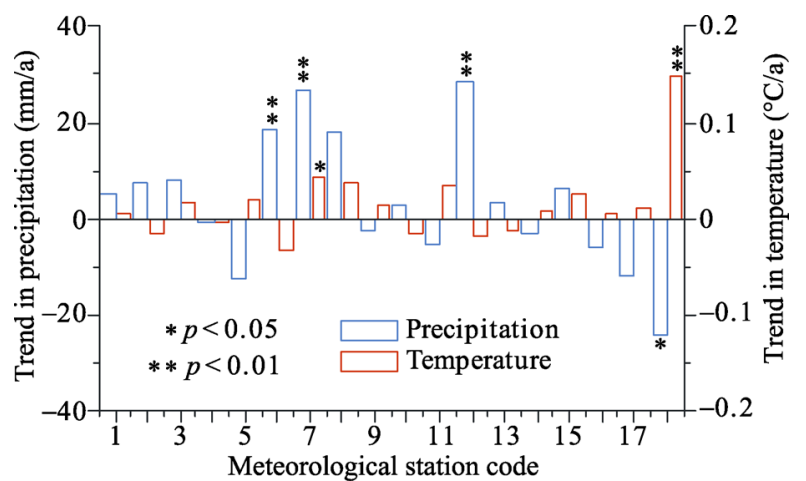

Figure 7 Trend of temperature and precipitation on the Qinba Mountains in 2000-2013 
tion time series data in 2000-2013. Results showed that the correlation of NDVI and temperature is $-0.012(-0.448$ to 0.228$)$, while the correlation of NDVI and precipitation is 0.612 ( -0.110 to 0.695$)$, through the significant level of 0.05 ; and stations 1 and 10 are both significantly correlated. Since the correlation of NDVI and precipitation is higher than that of NDVI and temperature, suggesting that vegetation growth in the study area is influenced to a greater extent by precipitation than by temperature.

Additionally, we further analyzed the variation in precipitation in the region. Considering that the vegetation coverage has shown a continuously decreasing trend since 2010, we focused on the changes of precipitation and vegetation after 2010. According to Figure 8a, there was a significant decline in precipitation in the central-eastern part, and only a limited area in the west showed growth due to rainfall. Moreover, regional average consecutive dry days (CDD) showed a significant increasing trend $(1.15 \mathrm{~d} / \mathrm{a})$ on the Qinba Mountains in 2000-2013, and the highest increase occurred between 2010 and 2013 (3.38 d/a). Stations of $3,5,7,10,13$, and 17 showed a notable increase in CDD, indicating an increase in drought in this area. Comparatively, vegetation growth showed a declining trend over a large extent at the same time of precipitation decrease, and thus water deficit may be responsible for the decrease in vegetation (Figure $8 \mathrm{~b}$ ). A previous study has reported that drought limits vegetation growth, lessens productivity, and eventually reduces the amount of fixed carbon in forests (Piao et al., 2014). Another study also documented that water use efficiency will be enhanced under drought conditions (Peñuelas et al., 2011). However, the water use efficiency in our study showed no obvious increase but a stable fluctuation in recent 15 years.
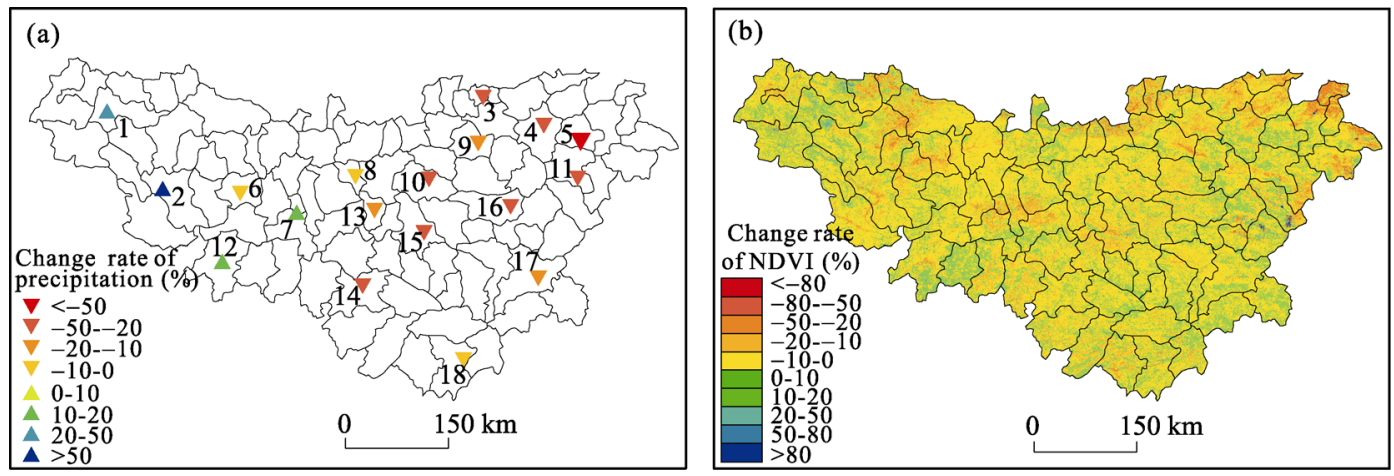

Figure 8 Precipitation deficit (a) and changes in the rate of NDVI (b) in 2010-2014

Climate fluctuation at a global scale results in extreme climate events and has a heavy impact on the terrestrial ecosystem. The results showed that the correlation between ENSO events and NDVI was $0.49(p<0.05)$ on the Qinba Mountains, suggesting that ENSO event has a significant influence on vegetation growth (Figure 9a). This finding is also consistent with the result of Hilker et al. (2014). To elucidate the relationship between vegetation growth and ENSO, we performed a composite analysis of NDVI and ENSO. First, we selected years with extreme El Nino (2002, 2006, and 2009) and La Nina (2007 and 2010), according to the statistics of El Nino and La Nina published by the National Climate Center, and then calculated the average NDVI in El Nino and La Nina years, respectively. As a result, years with extreme La Nina showed higher vegetation coverage than years with ex- 
treme El Nino (Figure 9b). According to the quantitative statistical results, in years with extreme El Nino, only $28.37 \%$ NDVI values showed a positive anomaly based on the average NDVI during the study period, while those that showed a negative anomaly were $71.63 \%$. On the contrary, in years with extreme La Nina, NDVI values that showed a positive anomaly were $80.48 \%$, while those that showed a negative anomaly were $19.52 \%$; this further confirmed that ENSO has a strong impact on vegetation growth. In order to justify the above phenomenon, we further analyzed precipitation in the region. The results show that in the years with extreme La Nina, higher precipitation was observed, which is beneficial for vegetation growth, while lower precipitation in the years with extreme El Nino restrains the growth of vegetation, especially in summer (Babst et al., 2013). In summary, climate fluctuation at a global scale has a huge influence on regional vegetation growth, and vegetation growth will be further threatened if there are more extreme events like drought in the future.
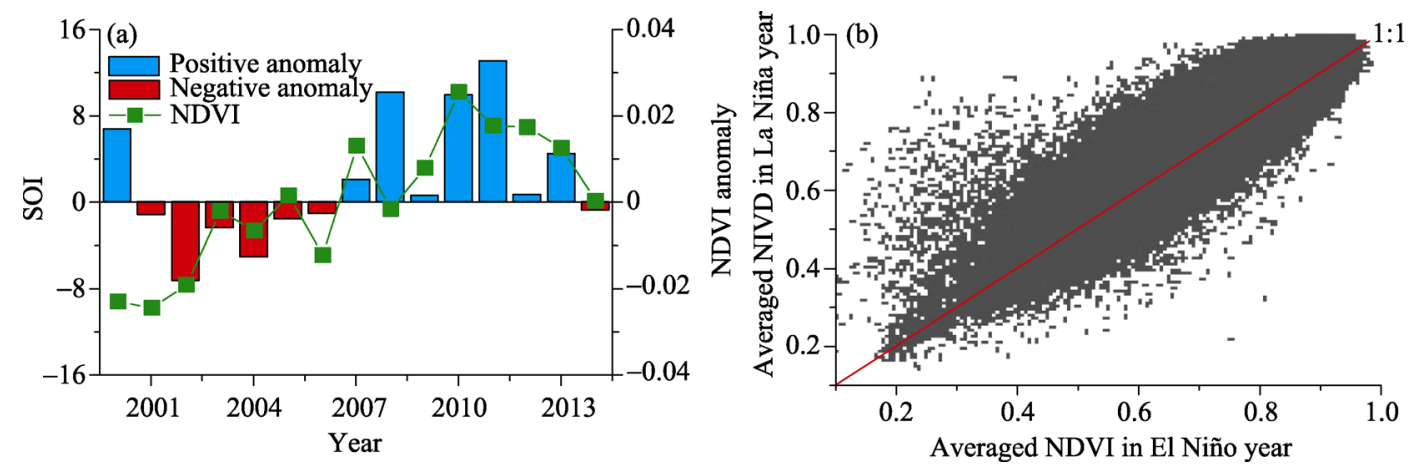

Figure 9 Correlations between NDVI and SOI

\subsection{Anthropogenic influences on vegetation dynamics}

In addition to the change in climate and atmospheric composition, human activity is also responsible for the change in vegetation growth, and it is divided into positive activities, such as ecological restoration projects like Grain for Green, and negative activities, such as expansion of cities and forest damage. Therefore, we performed the analysis on the basis of two aspects. First, we collected data for annual forestation area in 96 counties from the Statistic Yearbook of China Forestry (Figure 10a). According to the statistics, between 2002 and 2004, the highest afforestation and NDVI growth were observed, ecological projects caused a gradual increase in vegetation coverage; this proves that these projects are effective in improving the regional ecological environment. It should be noted that, between 2010 and 2014, vegetation coverage declined despite the restoration of the ecological environment, and extreme climate events like drought were most probably the reason. Second, on the basis of the exemplification of Hanzhong, we believe that city expansion will damage vegetation and reduce vegetation coverage. We calculated the rate of change in NDVI between 2010 and 2014 and then created 10 buffer circles with the center at the city and a radius of $2 \mathrm{~km}$ in order to perform quantitative analysis of city expansion on vegetation coverage. Furthermore, we evaluated the rate of change in every buffer circle and found out that vegetation coverage in circles around cities has decreased; this proves that the expansion of cities is responsible for the decline in vegetation coverage. It should be noted that although the expansion area is 
limited, the damage of the expansion on vegetation is greater than the occupation of the land alone.

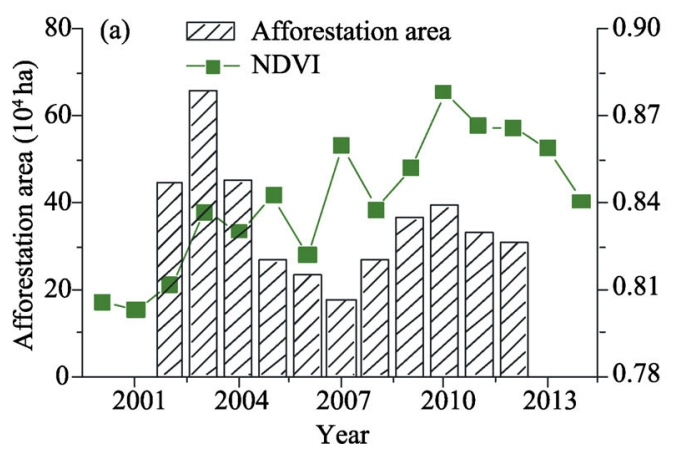

(b) Changes in vegetation in Hanzhong during 2010-2014

Figure 10 Influence of human activities on vegetation coverage

\section{Conclusions and discussion}

\subsection{Conclusions}

By using the MODIS-NDVI data and methods like trend analysis, Hurst exponent, and partial correlation analysis, we analyzed the variation in and factors that influenced vegetation coverage in the Qinba Mountain region. Our conclusions were as follows:

(1) Between 2000 and 2014, NDVI of the study area revealed a significant increase at an average speed of $2.8 \% / 10 \mathrm{a}$. However, a stable increase was detected before 2010 at the speed of $4.32 \% / 10 \mathrm{a}$, followed by a sharp decline at $-6.59 \% / 10 \mathrm{a}$.

(2) Vegetation coverage pattern was higher in the central part and lower at the edges. Higher values were observed in the Qinling and Daba Mountains in Shaanxi Province, and vegetation coverage increases with the increase in altitude until $3600 \mathrm{~m}$. NDVI declined rapidly afterwards.

(3) Vegetation coverage in the study area increased in general. The area with improved vegetation coverage was larger than the degraded area, being $81.32 \%$ and $18.68 \%$, respectively during the study period. Piecewise analysis revealed that $71.61 \%$ of the total study area showed a decreasing trend in vegetation coverage in 2010-2014.

(4) The reverse characteristics of vegetation coverage change were stronger than the same characteristics on the Qinba Mountains. About $46.89 \%$ of the entire study area is predicted to decrease in the future, while $34.44 \%$ of the total area will show a continuously increasing trend. The Hurst exponent showed little variation with the increase in altitude, and the value was generally less than 0.5 under $4500 \mathrm{~m}$.

(5) The change in vegetation coverage was mainly attributed to the deficit in precipitation. Meanwhile, ENSO has a large influence on vegetation growth, and composite analysis showed that vegetation coverage during the La Nina years was greater than that during the El Nino years.

(6) Human activities can induce ambiguous effects on vegetation coverage: both positive effects (through implementation of the ecological restoration project) and negative effects (through urbanization) were observed. 


\subsection{Discussion}

Currently, scholars worldwide have focused on the influence of extreme climate events on vegetation (Hilker et al., 2014; Zhou et al., 2014; Zhao et al., 2015). Since drought occurs over a large area and lasts for a long time, it is considered the most fatal threat to vegetation growth. In this study, we analyzed the effect of extreme events on vegetation growth in typical years with extreme climate conditions, and we discussed the relationship between ENSO and vegetation change. However, considering that vegetation responds differently to drought in different seasons, drought is not always the primary factor. Since the climate is relatively cool in winter and spring, temperature may be the main limiting factor for vegetation growth. As for summer and autumn, which are relatively hot, precipitation may be more important. Therefore, how vegetation responds to extreme climate in different seasons requires further study. Separation of the contribution of climate change and human activities on vegetation coverage change is still an issue. New creative methods and theories are required.

In this study, we only focused on the influence of climate on vegetation, and the feedback of vegetation to the regional climate, which is also an important research area about the relationship between vegetation and climate, was not included. In addition, the sensibility of vegetation to climate change under extreme climate conditions requires further analysis. Moreover, we only used MODIS-NDVI data, which is of the highest resolution, and we did not use multi-source NDVI datasets to verify the results. It should be noted that the results of vegetation coverage variation may not be the same because of the difference in resolution and quality of different NDVI datasets. Thus, further analysis of the consistency and uncertainty of variation evaluation of different NDVI datasets is required in order to obtain more robust results.

\section{References}

Babst Flurin, Poulter Benjamin, Trouet Valerie et al., 2013. Site- and species-specific responses of forest growth to climate across the European continent. Global Ecology and Biogeography, 22(6): 706-717.

Bai Hongying, 2014. The Response of Vegetation to Environmental Change in Qinba Mountains. Beijing: Science Press. (in Chinese)

Barati Susan, Rayegani Behzad, Saati Mehdi et al., 2011. Comparison the accuracies of different spectral indices for estimation of vegetation cover fraction in sparse vegetated areas. The Egyptian Journal of Remote Sensing and Space Science, 14(1): 49-56.

Compiling Committee of National Assessment Report on Climate Change, 2011. Second National Assessment Report on Climate Change. Beijing: Science Press. (in Chinese)

Ding Mingjun, Zhang Yili, Liu Linshan et al., 2007. The relationship between NDVI and precipitation on the Tibetan Plateau. Journal of Geographical Sciences, 17(3): 259-268.

Fu Bojie, 2014. The integrated studies of geography: Coupling of patterns and processes. Acta Geographica Sinica, 69(8): 1052-1059. (in Chinese)

Hilker Thomas, Lyapustin Alexeii, Tucker Comptonj et al., 2014. Vegetation dynamics and rainfall sensitivity of the Amazon. Proceedings of the National Academy of Sciences, 111(45): 16041-16046.

Holben Brentn, 1986. Characteristics of maximum-value composite images from temporal AVHRR data. International Journal of Remote Sensing, 7(11): 1417-1434.

Jiang Zhangyan, Huete Alfredor, Didan Kamel et al., 2008. Development of a two-band enhanced vegetation 
index without a blue band. Remote Sensing of Environment, 112(10): 3833-3845.

John Ranjeet, Chen Jiquan, Lu Nan et al., 2008. Predicting plant diversity based on remote sensing products in the semi-arid region of Inner Mongolia. Remote Sensing of Environment, 112(5): 2018-2032.

Kendall Mauricegeorge, 1948. Rank Correlation Methods. London: Charles Griffin \& Company Limited.

Lewis Simonl, Brando Paulom, Phillips Oliverl et al., 2011. The 2010 Amazon drought. Science, 331(6017): 554.

Liu Xianfeng, Ren Zhiyuan, Lin Zhihui et al., 2013. The spatial-temporal changes of vegetation coverage in the Three-River Headwater Region in recent 12 years. Acta Geographica Sinica, 68(7): 897-908. (in Chinese)

Liu Xianfeng, Zhu Xiufang, Pan Yaozhong et al., 2014. The spatial-temporal changes of cold surge in Inner Mongolia during recent 53 years. Acta Geographica Sinica, 69(7): 1013-1024. (in Chinese)

Liu Yinhan, 1983. Research on land category. Geography Central, (1): 47-56. (in Chinese)

Ma Zhihai, Peng Changhui, Zhu Qiuan et al., 2012. Regional drought-induced reduction in the biomass carbon sink of Canada's boreal forests. Proceedings of the National Academy of Sciences, 109(7): 2423-2427.

Nemani Ramakrishnar, Keeling Charlesd, Hashimoto Hirofumi et al., 2003. Climate-driven increases in global terrestrial net primary production from 1982 to 1999. Science, 300(5625): 1560-1563.

Parmesan Camille, 2006. Ecological and evolutionary responses to recent climate change. Annual Review of Ecology, Evolution, and Systematics, 637-669.

Peñuelas Josep, Canadell Josepg, Ogaya Romà, 2011. Increased water-use efficiency during the 20th century did not translate into enhanced tree growth. Global Ecology and Biogeography, 20(4): 597-608.

Piao Shilong, Nan Huijuan, Huntingford Chris et al., 2014. Evidence for a weakening relationship between interannual temperature variability and northern vegetation activity. Nature Communications, 5.

Reichstein Markus, Bahn Michael, Ciais Philippe et al., 2013. Climate extremes and the carbon cycle. Nature, 500(7462): 287-295.

Ren Zhiyuan, Li Jing, 2003. The valuation of ecological services from the vegetation ecosystems in the Qinling-Daba Mountains. Acta Geographica Sinica, 58(4): 503-511. (in Chinese)

Sen Pranabkumar, 1968. Estimates of the regression coefficient based on Kendall's tau. Journal of the American Statistical Association, 63(324): 1379-1389.

Shi Peijun, Kong Feng, Fang Jiayi, 2014. Spatio-temporal patterns of China decadal storm rainfall. Scientia Geographica Sinica, 34(11): 1281-1290. (in Chinese)

Shi Peijun, Sun Shao, Wang Ming et al., 2014. Climate change regionalization in China (1961-2010). Science China: Earth Sciences, 44(10): 2294-2306. (in Chinese)

Stocker T F, Qin D, Plattner G K et al., 2013. IPCC, 2013: Climate Change 2013: The Physical Science Basis. Contribution of Working Group I to the Fifth Assessment Report of the Intergovernmental Panel on Climate Change.

Walker B, Steffen W, 1997. IGBP Science No. 1: A Synthesis of GCTE and Related Research. Stockholm: IGBP: $1-24$.

Walther Gian-reto, Post Eric, Convey Peter et al., 2002. Ecological responses to recent climate change. Nature, 416(6879): 389-395.

Xu Jianhua, 2002. Mathematical Method in Modern Geography. 2nd ed. Beijing: Higher Education Press. (in Chinese)

Zhang Xuezhen, Dai Junhu, Ge Quansheng, 2013. Variation in vegetation greenness in spring across eastern China during 1982-2006. Journal of Geographical Sciences, 23(1): 45-56.

Zhao Zhiping, Wu Xiaopu, Li Guo et al., 2015. Drought in southwestern China and its impact on the net primary productivity of vegetation from 2009-2011. Acta Ecologica Sinica, 35(2): 1-16. (in Chinese)

Zhou Liming, Tian Yuhong, Myneni Rangab et al., 2014. Widespread decline of Congo rainforest greenness in the past decade. Nature, 509(7498): 86-90. 